\title{
Dissections of Regular Polygons into Triangles of Equal Areas
}

\author{
E. A. Kasimatis
}

Department of Mathematics, California State University, Sacramento, CA 95819, USA

Communicated by G. D. Chakerian

\begin{abstract}
This paper answers the question, "If a regular polygon with $n$ sides is dissected into $m$ triangles of equal areas, must $m$ be a multiple of $n$ ?" For $n=3$ the answer is "no," since a triangle can be cut into any positive integral number of triangles of equal areas. For $n=4$ the answer is again "no," since a square can be cut into two triangles of equal areas. However, Monsky showed that a square cannot be dissected into an odd number of triangles of equal areas.

We show that if $\boldsymbol{n}$ is at least 5 , then the answer is "yes." Our approach incorporates the techniques of Thomas, Monsky, and Mead, in particular, the use of Sperner's lemma and non-Archimedean valuations, but also makes use of affine transformations to distort a given regular polygon into one to which those techniques apply.
\end{abstract}

\section{Introduction}

We prove the following theorem:

Theorem. Let $n \geq 5$ be an integer. A regular $n$-gon is dissectable into $m$ triangles of equal areas if and only if $m$ is a multiple of $n$.

We exploit the methods introduced by Thomas [7] and Monsky [5] in the study of dissections of the square, and by Mead [4] in the study of dissections of the $n$-dimensional cube, and also make use of affine transformations.

\section{Background}

In 1967 Richman and Thomas [6] asked, "Can a rectangle be dissected into an odd number, $m$, of nonoverlapping triangles, all having the same areas?" Since 
affine transformations preserve combinatorial type, parallelness, and ratio of areas, "rectangle" can be replaced with "square" without loss of generality. Richman showed that the answer is "no" for $m=3$ or 5 and that if the answer were "yes" for some odd integer $m$, then it would also be "yes" for any odd integer larger than $m$.

Thomas [7] proved that the unit square cannot be dissected into an odd number of nonoverlapping triangles of equal areas if the coordinates of the vertices of the triangles are rational numbers with odd denominators. Monsky [5], extending Thomas's result, showed that the answer is always negative. His proof generalized Thomas's and consisted of two parts, one combinatorial and the other valuationtheoretic. The combinatorial argument generalized Sperner's lemma in the plane. By itself it could be used to extend the result to the case where the vertices of the triangles have rational coordinates without restricting the denominators to be odd. For the case of arbitrary vertices, Monsky extended the 2-adic valuation of $\mathbb{Q}$ to the reals, used it to partition the points in the plane into three sets, and then applied properties of valuations and a generalized Sperner's lemma.

In 1979 Mead [4] extended Monsky's valuation argument and his generalization of Sperner's lemma to $\mathbb{R}^{n}$, obtaining the following theorem:

An $n$-dimensional cube can be divided into $m$ n-dimensional simplices all of equal $n$-dimensional volumes if and only if $m$ is a multiple of $n !$.

We now describe these techniques in detail.

By a dissection of a polygon $P$ we mean a family of triangles whose union is $P$ and such that the intersection of the interiors of any two distinct triangles is empty. If, in addition, the triangles have the same areas, the dissection is called an equidissection of $P$. A polygon is equidissectable if it possesses an equidissection. A simplicial decomposition of $P$ is a dissection of $P$ such that the intersection of any two of the triangles is either empty, a vertex, or an entire side of both triangles.

Consider a dissection of $P$ in which each vertex of each triangle is labeled either $P_{0}, P_{1}$, or $P_{2}$. A triangle $T$ is complete if its vertices are labeled $P_{0}, P_{1}$, and $P_{2}$; a segment $S$ is complete if its vertices are labeled $P_{0}$ and $P_{1}$.

Lemma 1.1 (Sperner's Lemma, Simplicial $\mathbb{Z} / 2$ Form). Consider a simplicial decomposition of a polygon $P$ in which each vertex is labeled either $P_{0}, P_{1}$, or $P_{2}$. The number of complete triangles in the decomposition of $P$ is odd if and only if the number of complete segments on the boundary of $P$ is odd.

Though the segments labeled $P_{0} P_{1}$ appear to play a special role in Lemma 1.1, we could just as well have considered only segments of the form $P_{0} P_{2}$, or only segments of the form $P_{1} P_{2}$.

The following lemma generalizes the simplicial $\mathbb{Z} / 2$ form of Sperner's lemma to nonsimplicial dissections. (See [5].)

Lemma 1.2 (Sperner's Lemma, Nonsimplicial $\mathbb{Z} / 2$ Form). Consider a dissection of a polygon $P$ in which each vertex is labeled either $P_{0}, P_{1}$, or $P_{2}$. Suppose that no line contains vertices of all three types and that $P$ has an odd number of segments labeled $P_{0} P_{1}$ on its boundary. Then some triangle in the dissection is complete. 
Lemma 1.2 suffices for our method of proof of the theorem when $n$ is odd. However, for even $n$ we need a stronger form of Lemma 1.2.

Lemma 1.3 (Sperner's Lemma, Nonsimplicial Integral Form). Consider a dissection of a polygon $P$ in which each vertex is labeled either $P_{0}, P_{1}$, or $P_{2}$. Assume that no line contains vertices of all three types. Let $n_{1}\left(P_{0} P_{1} P_{2}\right)$ be the number of complete triangles for which the orientation given by the order $P_{0} P_{1} P_{2}$ is counterclockwise and let $n_{2}\left(P_{0} P_{1} P_{2}\right)$ be the number for which the orientation $P_{0} P_{1} P_{2}$ is clockwise. Orient the boundary of $P$ counterclockwise. Let $n_{1}\left(P_{0} P_{1}\right)$ be the number of complete segments on the boundary for which the order $P_{0} P_{1}$ is compatible with the orientation of the boundary and let $n_{2}\left(P_{0} P_{1}\right)$ be the number for which the orientation $P_{0} P_{1}$ is incompatible. Then

$$
n_{1}\left(P_{0} P_{1} P_{2}\right)-n_{2}\left(P_{0} P_{1} P_{2}\right)=n_{1}\left(P_{0} P_{1}\right)-n_{2}\left(P_{0} P_{1}\right)
$$

(In particular, if $n_{1}\left(P_{0} P_{1}\right) \neq n_{2}\left(P_{0} P_{1}\right)$, there is a complete triangle.)

The proof of Lemma 1.3 is similar to that of Lemma 1.2, and is left to the reader.

To find the parity of the number of complete segments on the boundary of $P$ in a labeling that meets the conditions of Lemma 1.2, it suffices to consider only the original edges of the polygon $P$. Since an edge of $P$ that contains points labeled $P_{0}$ and $P_{1}$ cannot contain points labeled $P_{2}$, the only edges of $P$ that can contain complete segments have vertices labeled $P_{0}$ and $P_{0}, P_{0}$ and $P_{1}$, or $P_{1}$ and $P_{1}$. By noting that the parity of the number of complete segments on any such edge is not altered by the introduction of points of type $P_{0}$ or $P_{1}$, we see that in a dissection of $P$ the number of complete segments on the boundary of $P$ is odd if and only if the number of complete edges of $P$ itself is odd.

The labels $P_{0}, P_{1}$, and $P_{2}$ will be assigned by using a function "ord", whose domain is $\mathbb{C}$ and whose range is contained in $\mathbb{R} \cup\{\infty\}$. Consider a fixed positive prime integer $p$. For any nonzero rational number $r / s,(r, s)=1$, define ord $(r / s)$ as follows: if $r / s=p^{m} \cdot r^{\prime} / s^{\prime}$, where $\left(p, r^{\prime}\right)=1=\left(p, s^{\prime}\right), \operatorname{ord}(r / s)=m$. Define $\operatorname{ord}(0)=\infty$. Then ord $: \mathbb{Q} \rightarrow \mathbb{Z} \cup\{\infty\}$ satisfies

(1) $\operatorname{ord}(x y)=\operatorname{ord}(x)+\operatorname{ord}(y)$,

(2) $\operatorname{ord}(x+y) \geq \min \{\operatorname{ord}(x), \operatorname{ord}(y)\}$, with equality if $\operatorname{ord}(x) \neq \operatorname{ord}(y)$,

(3) $\operatorname{ord}(x) \leq \infty$, with equality if and only if $x=0$.

This function can be extended to a function ord: $\mathbb{C} \rightarrow \mathbb{R} \cup\{\infty\}$ (in an infinite number of ways) which satisfies the same three conditions. (See [1] and [3].) We let "ord" denote one such function. Strictly speaking, this function should be denoted "ord ${ }_{p}$ ", but for the sake of simplicity we will generally omit the subscript $p$; the context will make it clear which prime $p$ is assumed.

Partition the points of the plane into three sets $P_{0}, P_{1}, P_{2}$ as follows:

$$
\begin{array}{lllll}
(x, y) \in P_{0} & \text { if } & \operatorname{ord}(x)>0 & \text { and } & \operatorname{ord}(y)>0, \\
(x, y) \in P_{1} & \text { if } & \operatorname{ord}(x) \leq 0 & \text { and } & \operatorname{ord}(x) \leq \operatorname{ord}(y), \\
(x, y) \in P_{2} & \text { if } & \operatorname{ord}(y) \leq 0 & \text { and } & \operatorname{ord}(y)<\operatorname{ord}(x) .
\end{array}
$$


For example, $(0,0)$ and $\left(p, p^{2}\right)$ are in $P_{0},(1,1),(1, p)$, and $(1 / p, 1)$ are in $P_{1}$, and $(p, 1 / p)$ and $(1,1 / p)$ are in $P_{2}$.

The key property of this labeling is:

Lemma 1.4. For any complete triangle $T$, relative to the labeling induced by ord,

$$
\operatorname{ord}(2 \cdot \operatorname{area} T) \leq 0 \text {. }
$$

For a proof, see [5].

It follows from Lemma 1.4 that the labeling defined by the partition of the plane into the sets $P_{0}, P_{1}, P_{2}$ satisfies the hypotheses of Lemmas 1.2 and 1.3. For if a line contained points labeled $P_{0}, P_{1}, P_{2}$, then the degenerate complete triangle they would form would have area 0 , which contradicts Lemma 1.4.

For any dissection of a polygon $P$, we say that the labeling is complete if the number of complete edges on the boundary of $P$ is odd. In this case there is an odd number of complete segments on the boundary, so some triangle $T$ is complete. Suppose a polygon $P$ is equidissected into $m$ triangles and that the labeling of $P$ is complete, or, more generally, that there is a complete triangle $T$ in the equidissection. Then

$$
0 \geq \operatorname{ord}(2 \cdot \operatorname{area} T)=\operatorname{ord}(2 \cdot \operatorname{area} P)-\operatorname{ord}(m)
$$

and therefore

$$
\operatorname{ord}(m) \geq \operatorname{ord}(2 \cdot \text { area } P)
$$

This inequality is the key to obtaining information about $m$.

\section{The Proof}

We illustrate the general argument by showing that if a regular hexagon is equidissected into $m$ triangles then 6 divides $m$. Let $T$ be the affine transformation which maps three consecutive vertices of the hexagon to the points $(0,1),(0,0)$, and $(1,0)$. It follows that the center of the hexagon is mapped to $(1,1)$ while the other three vertices go to $(2,1),(2,2)$, and $(1,2)$. Let $P^{*}$ be the image hexagon. Then $P^{*}$ has area 3 and admits an equidissection into $m$ triangles.

To show that 3 divides $m$, consider an ord for $p=3$ and label the plane as in Section 1 . Since the edge joining $(0,0)$ and $(1,0)$ is the only complete edge on the boundary of $P^{*}$, the labaling of $P^{*}$ is complete, and, by Lemma 1.2 , there is a complete triangle. Therefore, ord $m \geq \operatorname{ord}\left(2 \cdot\right.$ area $\left.P^{*}\right)=1$, and 3 divides $m$.

Now consider an ord for $p=2$ and again label the plane as in Section 1. Then the vertices of $P^{*}$, taken in counterclockwise order from the origin, are of types $P_{0}, P_{1}, P_{2}, P_{0}, P_{1}, P_{2}$; so the labeling of $P^{*}$ is not complete. Nevertheless, by Lemma 1.3, there are at least two complete triangles in the equidissection of $P^{*}$.

The treatment of the equidissection of a regular $n$-gon $(n \geq 5)$ into $m$ triangles 
follows a similar pattern. We show that $n$ divides $m$ by proving that $\operatorname{ord}_{p}(m)>$ $\operatorname{ord}_{p}(n)-1$ for each prime $p$ dividing $n$.

Fix such a prime $p$. Set $\theta=2 \pi / n$ and $\zeta=e^{i \theta}$.

Let $P$ be the regular $n$-gon with vertices at $((\cos j \theta)-1, \sin j \theta), j \in \mathbb{Z} / n$. Let $T$ be the linear map

$$
(x, y) \rightarrow-2\left(x \cos \frac{\theta}{2}-y \sin \frac{\theta}{2}, x \cos \frac{\theta}{2}+y \sin \frac{\theta}{2}\right),
$$

and let $P^{\prime}$ be the image of $P$ under this map. Since $P$ is inscribed in a circle of radius $1,2 \cdot$ area $P=n \sin \theta$ and $2 \cdot$ area $P^{\prime}=(n \cdot \sin \theta)$ det $T=n \cdot(2 \sin \theta)^{2}$. The image of $(\cos u, \sin u)$ under $T$ is $(-2 \cos (u+\theta / 2),-2 \cos (u-\theta / 2))$. Since $-2(\cos (A+B)-\cos (A-B))=4 \sin A \sin B$, the image under $T$ of $((\cos u)-$ $1, \sin u)$ is $(4 \sin ((u+\theta) / 2) \sin (u / 2), 4 \sin ((u-\theta) / 2) \sin (u / 2))$. In particular, the vertices of $\boldsymbol{P}^{\prime}$ are the points

$$
\left(x_{j}, y_{j}\right)=\left(4 \sin \frac{(j+1) \theta}{2} \sin \frac{j \theta}{2}, 4 \sin \frac{(j-1) \theta}{2} \sin \frac{j \theta}{2}\right) .
$$

Since $2 \cdot \sin (j \theta / 2)$ is the product of $1-\zeta^{j}$ by a root of unity, we find that $\operatorname{ord}(2 \cdot \sin (j \theta / 2))=\operatorname{ord}\left(1-\zeta^{j}\right)$ and therefore that

$$
\operatorname{ord}\left(x_{j}\right)=\operatorname{ord}\left(1-\zeta^{j}\right)+\operatorname{ord}\left(1-\zeta^{j+1}\right)
$$

while

$$
\operatorname{ord}\left(y_{j}\right)=\operatorname{ord}\left(1-\zeta^{j}\right)+\operatorname{ord}\left(1-\zeta^{j-1}\right)
$$

We use the following well-known facts from the theory of cyclotomic fields. If $p$ is odd and $\zeta^{j} \neq 1$, then ord $\left(1-\zeta^{j}\right) \leq 1 /(p-1)$ with equality if and only if $\zeta^{j}$ is a $p$ th root of unity. If $p=2$ and $\zeta^{j} \neq \pm 1$, then ord $\left(1-\zeta^{j}\right) \leq \frac{1}{2}$ with equality if and only if $\zeta^{j}= \pm i$. (See [2].)

Lemma 2.1. For $j \in \mathbb{Z} / n, n \geq 4$, ord ${ }_{p}\left(y_{j}\right)<1$ whenever

(a) $p$ is odd and $j \neq 0$ or 1 , or

(b) $p=2$ and $j \neq 0,1, n / 2$, or $n / 2+1$.

Proof. Assume that $p$ is an odd prime divisor of $n$. Since neither $\zeta^{j}$ nor $\zeta^{j-1}$ is $1, \operatorname{ord}\left(y_{j}\right) \leq 2 /(p-1)$. The result follows unless $p=3$ and $\zeta^{j}$ and $\zeta^{j+1}$ are both cube roots of unity. However, this is the case $n=3$, which is excluded. Assume now that $p=2$ and $j \neq 0,1, n / 2$, or $n / 2+1$. Then neither $\zeta^{j}$ nor $\zeta^{j-1}$ is \pm 1 . Tnus, we obtain the result unless $\zeta^{j}$ and $\zeta^{j-1}$ are each $i$ or $-i$, which is impossible.

For $p$ odd, fix $k \in \mathbb{Z} / n, k \neq 0$ or 1 , so that ord $\left(y_{k}\right)$ is as large as possible. For $p=2$ fix $k \in \mathbb{Z} / n, k \neq 0,1, n / 2$, or $n / 2+1$, so that ord $\left(y_{k}\right)$ is as large as possible. In both cases, let $P^{*}$ be the image of $P^{\prime}$ under the linear map $(x, y) \rightarrow\left(x / x_{1}, y / y_{k}\right)$. 
Lemma 2.2. $\operatorname{ord}\left(2 \cdot\right.$ area $\left.P^{*}\right)>\operatorname{ord}(n)-1$.

Proof. Observe that

$$
\begin{aligned}
\operatorname{ord}\left(2 \cdot \operatorname{area} P^{*}\right) & =\operatorname{ord}\left(2 \cdot \operatorname{area} P^{\prime}\right)-\operatorname{ord}\left(x_{1}\right)-\operatorname{ord}\left(y_{k}\right) \\
& =\operatorname{ord}(n)+2 \operatorname{ord}\left(1-\zeta^{2}\right)-\left(\operatorname{ord}(1-\zeta)+\operatorname{ord}\left(1-\zeta^{2}\right)\right)-\operatorname{ord}\left(y_{k}\right) \\
& =\operatorname{ord}(n)+\operatorname{ord}(1+\zeta)-\operatorname{ord}\left(y_{k}\right) \\
& \geq \operatorname{ord}(n)-\operatorname{ord}\left(y_{k}\right) .
\end{aligned}
$$

But Lemma 2.1 shows that $\operatorname{ord}\left(y_{k}\right)<1$.

Now label the points in the plane as in Section 1 . Note that $P^{*}$, like $P$, has an equidissection into $m$ triangles.

Lemma 2.3. Some triangle in an equidissection of $P^{*}$ is complete.

Proof. Assume first that $p$ is odd. We show that the labeling of $P^{*}$ is complete by showing that the boundary of $p^{*}$ contains only one complete edge. Now $\left(x_{0} / x_{1}, y_{0} / y_{k}\right)=(0,0)$ and $\left(x_{1} / x_{1}, y_{1} / y_{k}\right)=(1,0)$ are of types $P_{0}$ and $P_{1}$, respectively. Since $x_{-1}=0$ and ord $\left(y_{k}\right) \geq \operatorname{ord}\left(y_{-1}\right),\left(x_{-1} / x_{1}, y_{-1} / y_{k}\right)$ is of type $P_{2}$. Furthermore, if $j \in \mathbb{Z} / n, j \neq-1,0$, or 1 , then $\operatorname{ord}\left(y_{j} / y_{k}\right) \leq 0$ and $\left(x_{j} / x_{1}, y_{j} / y_{k}\right)$ cannot be of type $P_{0}$. It follows that the edge joining the points $(0,0)$ and $(1,0)$ is the only complete edge on the boundary of $P^{*}$. This proves that the labeling of $P^{*}$ is complete; hence, there is at least one complete triangle in an equidissection of $P^{*}$.

Assume now that $p=2$, and set $r=n / 2$. Let $C=(u, v)$ be the center of $P^{*}$. We claim that $2 C$ is a point of type $P_{0}$ or, in other words, that ord $(2 u)>0$ and $\operatorname{ord}(2 v)>0$. Now the center of $P^{\prime}$ is $(a, a)$ with $a=2 \cos (\theta / 2)=(1+\zeta)$ (root of unity); the center of $P^{*}$ is $\left(a / x_{1}, a / y_{k}\right)$. Since ord $\left(x_{1}\right)=\operatorname{ord}\left(y_{2}\right) \leq \operatorname{ord}\left(y_{k}\right)<1$, the result follows. Observe that if $Q$ and $Q^{\prime}$ are opposite vertices of $P^{*}, Q+Q^{\prime}=2 C$ is of type $P_{0}$; it follows that $Q$ and $Q^{\prime}$ are of the same type. In the paragraph above, we saw that when $j \in \mathbb{Z} / n$ is $-1,0$, and 1 , then the corresponding vertices $\left(x_{j} / x_{1}, y_{j} / y_{k}\right)$ of $P^{*}$ are of types $P_{2}, P_{0}$, and $P_{1}$, respectively. It follows that when $j=r-1, r$, and $r+1$, the corresponding vertices are again of types $P_{2}, P_{0}$, and $P_{1}$, respectively.

Suppose finally that $j \in \mathbb{Z} / n, j \neq-1,0,1, r-1, r$, or $r+1$. Then $\operatorname{ord}\left(y_{j} / y_{k}\right) \leq 0$ and the vertex $\left(x_{j} / x_{1}, y_{j} / y_{k}\right)$ of $P^{*}$ cannot be of type $P_{0}$. Therefore, $n_{1}\left(P_{0} P_{1}\right)-$ $n_{2}\left(P_{0} P_{1}\right)=2$. As in the case of the regular hexagon, by Lemma 1.3 , there are at least two complete triangles in an equidissection of $P^{*}$.

The proof of the theorem follows immediately. Let $p$ be a prime divisor of $n$. Combining the remarks after Lemma 1.4 with Lemmas 2.2 and 2.3, we find that $\operatorname{ord}(m) \geq \operatorname{ord}\left(2 \cdot\right.$ area $\left.P^{*}\right)>\operatorname{ord}(n)-1$. Since ord $(m)$ and ord $(n)$ are integers, it follows that $\operatorname{ord}(m) \geq \operatorname{ord}(n)$. As this is true for all primes $p$ dividing $n, m$ must be a multiple of $n$. 
Remark. In its initial form this proof used the $\mathbb{Z} / 2$ form of Sperner's lemma, which is strong enough to establish the theorem for odd $n$. However, for even $n$, this form of Sperner's lemma establishes only that in an equidissection of a regular $n$-gon into $m$ triangles, $m$ is a multiple of $n / 2$. I wish to thank one of the referees for pointing out that the integral version of Sperner's lemma disposes of even $n$ as well.

\section{References}

1. G. Bachman, Introduction to p-adic Numbers and Valuation Theory, Academic Press, New York, 1964.

2. H. Hasse, Number Theory, Springer-Verlag, Berlin, 1980.

3. K. Ireland and M. Rosen, A Classical Introduction to Modern Number Theory, Springer-Verlag, New York, 1982.

4. D. G. Mead, Dissection of the hypercube into simplexes, Proc. Amer. Math. Soc., 76 (1979), 302-304.

5. P. Monsky, On dividing a square into triangles, Amer. Math. Monthly, 77 (1970), 161-164.

6. F. Richman and J. Thomas, Problem 5479, Amer. Math. Monthly, 74 (1967), 329.

7. J. Thomas, A dissection problem, Math. Mag., 41 (1968), 187-190.

Received September 17, 1986, and in revised form November 18, 1987. 